\title{
A micro-capacitive pressure sensor design and modelling
}

\author{
Ali E. Kubba ${ }^{1}$, Ahmed Hasson ${ }^{1}$, Ammar I. Kubba ${ }^{2}$, and Gregory Hall ${ }^{1}$ \\ ${ }^{1}$ Fusion Innovations Ltd., Research and Innovation Services, Birmingham Research Park, Vincent Drive, \\ Edgbaston, Birmingham, B15 2SQ, UK \\ ${ }^{2}$ School of Engineering, Mechanical Engineering, University of Birmingham, \\ Edgbaston, Birmingham, B15 2TT, UK \\ Correspondence to: Ali E. Kubba (a.e.s.kubba@bham.ac.uk)
}

Received: 19 October 2015 - Revised: 5 February 2016 - Accepted: 15 February 2016 - Published: 30 March 2016

\begin{abstract}
Measuring air pressure using a capacitive pressure sensor is a robust and precise technique. In addition, a system that employs such transducers lies within the low power consumption applications such as wireless sensor nodes. In this article a high sensitivity with an elliptical diaphragm capacitive pressure sensor is proposed. This design was compared with a circular diaphragm in terms of thermal stresses and pressure and temperature sensitivity. The proposed sensor is targeted for tyre pressure monitoring system application. Altering the overlapping area between the capacitor plates by decreasing the effective capacitance area to improve the overall sensitivity of the sensor $(\Delta C / C)$, temperature sensitivity, and built-up stresses is also examined in this article. Theoretical analysis and finite element analysis (FEA) were employed to study pressure and temperature effects on the behaviour of the proposed capacitive pressure sensor. A MEMS (micro electro-mechanical systems) manufacturing processing plan for the proposed capacitive sensor is presented. An extra-low power short-range wireless read-out circuit suited for energy harvesting purposes is presented in this article. The developed read-out circuitry was tested in terms of sensitivity and transmission range.
\end{abstract}

\section{Introduction}

This article presents a proposed design of a MEMS capacitive pressure sensor for tyre inflation pressure measurement purposes. A diaphragm-based pressure sensing mechanism is employed and analysed in both analytical and numerical techniques, including Matlab and finite element analysis (FEA), which are employed to examine the proposed micro-pressure sensor response under a certain range of pressure and temperature conditions. A low power read-out and transmission circuit for the capacitive pressure sensor is also illustrated.

\subsection{Motivation}

This research is part of a TSB funded project which is led by Fusion Innovations Ltd. ECORR (Variable Rolling Resistance Tyre System) is an Innovate UK collaborative project as part of the Low Carbon Vehicles Disruptive technologies field. This project is currently partnered by Randle Engineering, Potenza Technology, and the University of Birmingham ${ }^{1}$.

According to the Pressure Sensor Market report (MarketsandMarkets, 2015) published by MarketsandMarkets, the global pressure sensor market was valued at USD 6.7 billion in 2014 and is expected to grow to USD 9.48 billion by 2020 . In 2014, around $36.7 \%$ of commercial pressure sensors were piezoresistive, followed by capacitive pressure sensors as the second dominant type, accounting for a market share of $27.6 \%$. The automotive industry is the largest application market for pressure sensors and accounted for a $25 \%$ share of the total revenue of 2014 (MarketsandMarkets, 2015). Sizable growth of the pressure sensor market in the Asia-Pacific region, particularly in the automotive industry, is predicted due to the introduction of new regulations and legislations re-

\footnotetext{
${ }^{1}$ Fusion Innovations Ltd, available at: http://www. fusion-innovations.com/, last access: 30 December 2015.
} 


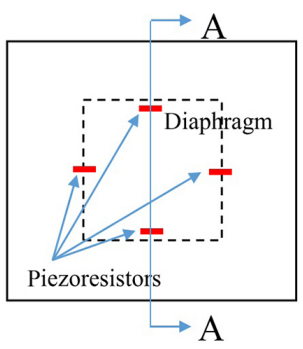

(a)

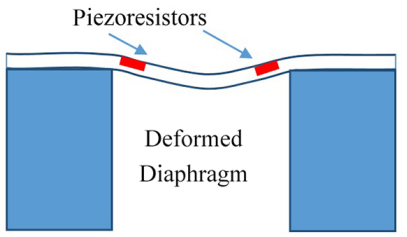

(b)
Figure 1. Typical piezoresistive pressure sensor structure. (a) Top view of the piezoresistive pressure sensor. Four piezoresistors are placed on each edge forming a Wheatstone bridge circuit. (b) Cross section A-A showing deflected diaphragm with piezoresistors at maximum stress locations (Barlian et al., 2009).

garding the use of such sensors in vehicles. More TPMS (tyre pressure monitoring system) market analysis can be found in Technavio (2015).

A listing for pressure sensors, manufacturers, and a technical comparison between both piezoresistive and capacitive pressure sensors can be found in Fragiacomo (2012).

\subsection{Piezoresistive pressure sensors}

Piezoresistive pressure sensors are based on the piezoresistive effect, which is a change in resistance with the applied stress. By applying piezoresistor(s), with known values and where a maximum variation of stresses occurs, to a pressure sensitive diaphragm, and monitoring the change in that resistor as the applied pressure varies, in most cases the change in the applied pressure can be detected by using a Wheatstone bridge circuit. The fact that singlecrystal silicon has a piezoresistive nature, and that silicon is mainly used in micro-machining, enabled production of semi-conductor-based sensors (Eaton and Smith, 1997). Examples of industrial piezoresistive pressure sensors are shown in Figs. 1 and 2.

Piezoresistive pressure sensors have the advantages of low cost, linearity, a simple read-out circuit, and the possibility of being batch produced. The piezoresistive coefficients of each mounted resistor depend upon the orientation of the wafer and diaphragm, the type and amount of doping, and the temperature (Beeby et al., 2004). The latter is a main disadvantage in applying piezoresistive pressure sensors to tyre monitoring systems, as tyre temperature changes over a wide range. Power consumption is highly related to the sizes of the mounted resistors' values, which vary according to the size of the piezoresistive pressure sensor overall. However, most existing commercially available TPMSs include a piezoresistive pressure sensor for their relatively low price. A typical commercial piezoresistive pressure sensor developed by Intersema Sensoric, a Measurement Specialties company (2009), is shown in Fig. 3.
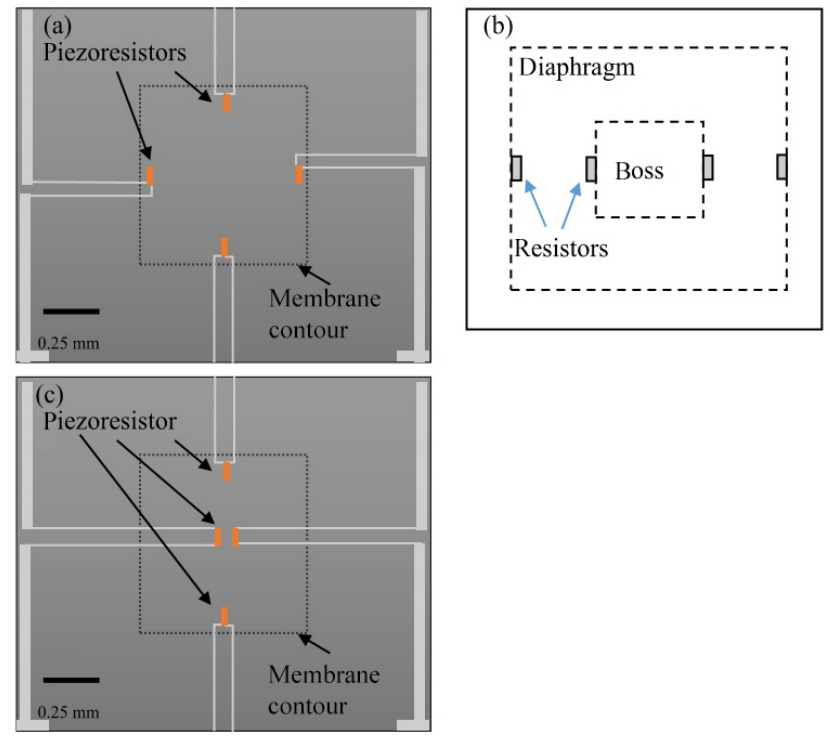

Figure 2. Resistor placement on diaphragm-based piezoresistive pressure sensors: (a) four resistors placed on the diaphragm edges and (b) two resistors on the diaphragm edges and the other two at the centre of the diaphragm. In both cases, the resistors are connected in Wheatstone bridge (c) resistors mounted on the inner and outer perimeters on a bossed diaphragm (Beeby et al., 2004; ChienHung et al., 2006).

Scaling down the mounted resistors creates an increase in power consumption - providing the same piezoresistive coefficient, mounting accuracy, and noise problems (Cullinan et al., 2012). In addition, the limitation of the resistors' size might reduce the sensitivity of the piezoresistive pressure sensor by averaging the developed stress on the sensor diaphragm. Another problem with piezoresistive pressure sensors is that piezoresistance is susceptible to junction leakage and surface contamination (Bao, 2000). This is true when using silicon as the main substrate to fabricate a MEMS pressure sensor, and can be overcome by using SOI (silicon on insulator) instead (Li Sainan et al., 2015), though it would double the sensor's substrate cost. A good review of the working principles and sensitivity of piezoresistive pressure sensors is explained in Gad-el-Hak (2002). With capacitive and piezoresistive measurement principles, there are competing concepts on the market. Whereas the piezoresistive pressure sensor is found in the vast majority of commercial TPMS (Fragiacomo, 2012; Nwagboso, 2012), it does not meet the requirement of battery-less systems anymore due to the fact that the power consumption is much higher, and since sleep modes for measurement acquisition are mandatory (Thurau and Ruohio, 2004). 


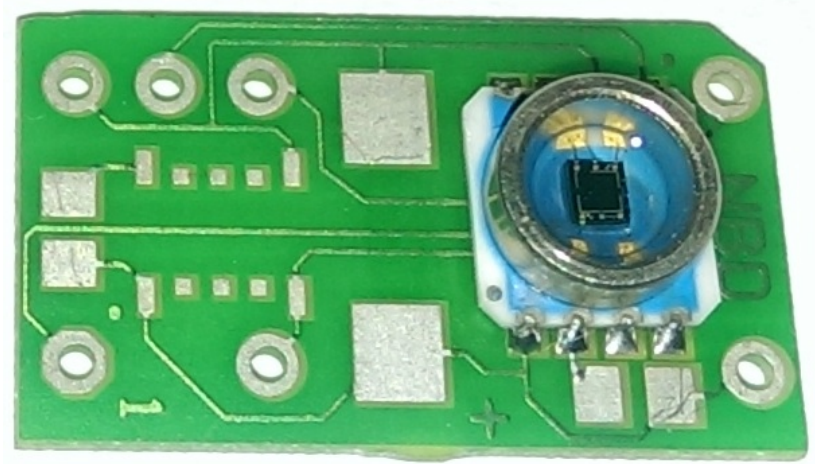

Figure 3. Miniature SMD (surface-mounted device) pressure sensor mounted on a read-out PCB (printed circuit board) developed by Intersema Sensoric (now Measurement Specialties, Inc., a TE Connectivity company; photo taken with permission from Measurement Specialties).

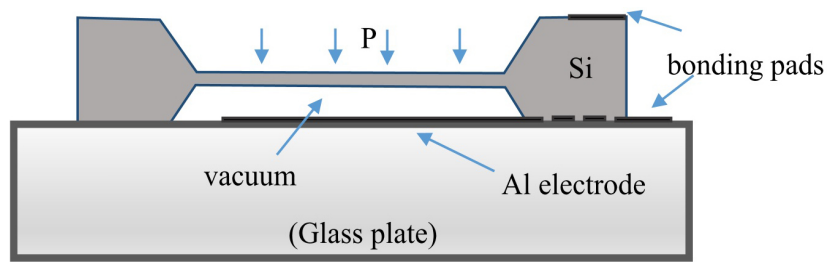

Figure 4. A schematic view of a basic capacitive pressure transducer (Bao, 2000).

\subsection{Capacitive pressure sensors}

This type of micro-machined pressure sensor was first developed in the late 1970s and early 1980s (Gad-el-Hak, 2002). It can be classified as the simplest in principle among all pressure sensing mechanisms. Capacitive pressure sensors primarily consist of two parallel conductive plates, so-called electrodes, separated by a dielectric material. Usually one of the electrodes is pressure sensitive, whereas the other electrode is located on a rigid substrate beneath it. However, a capacitive pressure sensor with two sensitive diaphragms has been developed (Fonseca et al., 2002). When pressure is applied onto the sensitive diaphragm, the cavity enclosed between the two parallel plates reduces in volume as the sensitive diaphragm deflects and approaches the stationary one, resulting in a detectable change in the capacitance between the electrodes. A schematic diagram for a typical capacitive pressure sensor is shown in Fig. 4.

The principle advantages of capacitive pressure sensors over piezoresistive pressure sensors are lower power consumption, increased pressure sensitivity, and lower temperature cross-sensitivity (Lee and Wise, 1982; Eaton and Smith, 1997; Eddy and Sparks, 1998; Bever et al., 2003; Beeby et al., 2004). In addition, because there is no need to mount any resistors on the sensor diaphragm, scaling down the device dimensions is easier because concerns about stress averaging and resistor tolerance are eliminated. Variation of temperature has a major contribution to the sensitivity of piezoresistive pressure sensors, mainly due to the resistance dependence on temperature. This factor is eliminated in capacitive sensors (Gad-el-Hak, 2002). There is virtually no power consumption in the sensing element in capacitive pressure sensors due to the dc current component being zero (Gadel-Hak, 2002) and the low power capability of the capacitive measuring principle (Kolle et al., 2004).

Capacitive pressure sensors have been applied to TPMS devices due to their low power consumption, high accuracy and low temperature sensitivity (Rudolf and Hoogerwerf, 1997; APOLLO, 2003; Thurau and Ruohio, 2004; Kolle et al., 2004). Bracke et al. (2007) presented the power consumption of various ultra-low power capacitive sensors' interfaces and showed experimentally that it is possible to achieve an average power consumption of $7.3 \mu \mathrm{W}$ for a $10 \mathrm{~Hz}$ sampling frequency and 8 bit accuracy in the 100 to $130 \mathrm{kPa}$ pressure range. This amount of power consumption is lower than the average piezoresistive pressure sensor power consumption if a similar sampling rate and digitalization level is used. A new patented capacitive sensor measurement chip (PicoCap $)^{2}$ could be utilized for TPMS devices as it offers signal digitalization at a high sampling rate (up to $500 \mathrm{k} \mathrm{sample} \mathrm{s}^{-1}$ ) under an extra low power requirement.

The main limitations of capacitive pressure sensors in general are nonlinearity between the applied pressure, the change in capacitance, and the large impedance of the sensor output signal (Tian et al., 2009), which also affects the interface circuit design, and the parasitic capacitance between the interface circuit and the device output can have a significant negative impact on the read-out, which means that the circuit must be placed in close proximity to the device in a hybrid or monolithic implementation. To address the nonlinearity problem in capacitive pressure sensors, different approaches have been used to mitigate this drawback, e.g. bossed diaphragms, centrally clamped diaphragms and partial electrode patterned diaphragms; however, these approaches reduce the overall sensor sensitivity and limit the measured pressure range. Touch mode capacitive pressure sensors, as shown in Fig. 5, are commonly used to employ linearity as regards the nonlinearity problems in ordinary capacitive pressure sensors. One potential drawback of touch mode devices is hysteresis arising from friction between the surfaces as they move together and apart, as well as the risk of stiction (Beeby et al., 2004).

Another important issue in the making of capacitive pressure sensors is sealing, which is a fabrication process complexity (Tian et al., 2009). Extra care should be taken when fabricating and packaging absolute pressure sensors and especially in sealing the vacuum cavity beneath the sensitive diaphragm, as it is the reference pressure for the sensor (Gad-

\footnotetext{
2 acam messelektronik gmbh, available at: http://www.acam.de/ de/produkte/picocap/, last access: 8 October 2015.
} 


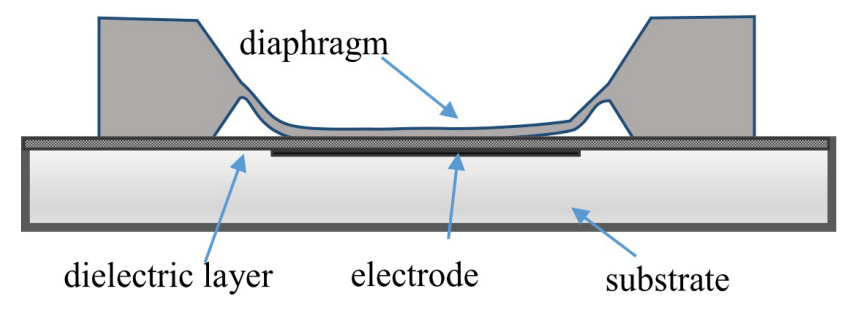

Figure 5. A touch mode capacitive pressure sensor (Gad-el-Hak, 2002).

el-Hak, 2002). Proper sealing in absolute capacitive pressure sensors is crucial to achieve long term stability. Due to cost issues in both wafers and fabrication processes, and the match between Pyrex glass and silicon thermal expansion coefficients, Pyrex glass substrate is a common material for the sensor constraint base when making capacitive pressure sensors to support the sensor silicon dies. Anodic bonding is usually used to attach Pyrex glass to silicon wafers, which is essential to produce a reliable and hermetically sealed MEMS capacitive pressure sensor under medium bonding temperature (Frank, 2000; Hsu, 2008). Glass is therefore a well-known material, but also has some specific characteristics like outgasing and absorption of residual gas on the glass surface which would lead to a change in the internal pressure. In order to address this problem, an interesting approach was used in fabricating a capacitive pressure sensor - which is especially designed for TPMS applications, as shown in Thurau and Ruohio (2004). In this design, a wet etched vacuum reservoir, also called a gas pocket, was added and connected to the reference pressure volume underneath the sensor diaphragm enabling good long term stability. A good review of a MEMS capacitive pressure sensor, using a technology developed by a group of engineers in Finland who originally developed pressure sensors targeted for weather measurement application, and which were especially designed to withstand harsh environment conditions, is explained in detail in Thurau and Ruohio (2004). A comparison of various high-temperature pressure sensors and passive wireless pressure sensors is well presented by Fonseca (2007).

\subsection{Resonant pressure sensors}

Resonance micro-machined pressure sensors were first developed in the early 1980s by Greenwood (1984). The resonant sensing mechanism is based on containing a resonant structure in which its resonance frequency is a function of a mechanical quantity, such as pressure, strain, temperature, etc. This type of sensor can be considerably more robust than the measurement of a resistor or capacitor, and therefore it is mainly affected by the mechanical qualities rather than electrical qualities of the device (Gad-el-Hak, 2002).

The main advantages of this type of sensing technique over capacitive and piezoresistive techniques are its high accuracy and quality factors. In addition, resonant pressure sensors' output is frequency; therefore, interfacing to a digital system can be easier. However, the fabrication of such sensors has some technical challenges. More details about the advantages and disadvantages are explained in Gad-el-Hak (2002) and Beeby et al. (2004). Other main issues with resonant pressure sensors are power consumption, which is considerably higher than conventional capacitive and pressure sensors, and temperature sensitivity, which affects the resonator structure material properties and geometry, and therefore changes its natural frequency.

Having vehicle tyre inflation pressure measurement, powered by energy harvesting, as the main thrust in this work, the dynamic environment of the measurement system forces onto the system a wide range of random vibration excitation, mainly from tyre rolling (Tsujiuchi et al., 2005; Brusarosco et al., 2008, 2010), and also from various vehicle components or vibrations transferred through the road originating from other vehicles or nearby machinery and high-impact and centrifugal acceleration components (APOLLO, 2005), which means that the pressure measurement system must not be sensitive to or affected by such conditions. This is particularly a major problem when using a resonant sensing system, as it is not possible to isolate the tyre pressure sensing system from all the vibrations occurring within the tyre structure. For this reason, and due to the high power consumption of resonant pressure sensors, this type of sensor is out of the scope of this research. However, Grossmann (1999) claims that developing a quartz-based resonant passive tyre pressure sensor, in which its natural frequency lies in the megahertz range, is extremely stable. On the other hand, the system is battery free and powered by an RF signal generated by the read-out system, which has to be mounted within a short distance from the resonator and is powered by the vehicle battery and consumes $10 \mathrm{~mW}$ of power. It is not clear whether the resonator was tested in a moving vehicle. Hannan et al. (2008) state that this type of tyre pressure sensor has a main disadvantage of low robustness in a harsh environment during vehicle operation.

Preliminary resonant devices are rather complex in terms of design, fabrication, and packaging. In addition, resonant devices contain piezoresistors for strain measurements and piezoelectric materials for vibration excitation, either of which can be used directly in the sensing structure for measuring most mechanical quantities. As such, using the resonant approach in the sensing mechanism is only justified in high-performance sensing applications (Beeby et al., 2004). An example of a commercialized resonant pressure sensor, developed by Druck Limited, is shown in Fig. 6 below (Beeby et al., 2004; Druck, 2012).

In the previous paragraphs, the three most commercially available micro-machined pressure sensors were explained and compared. In the following paragraphs within this section, other pressure sensing techniques are briefly covered (Gad-el-Hak, 2002; Beeby et al., 2004). 

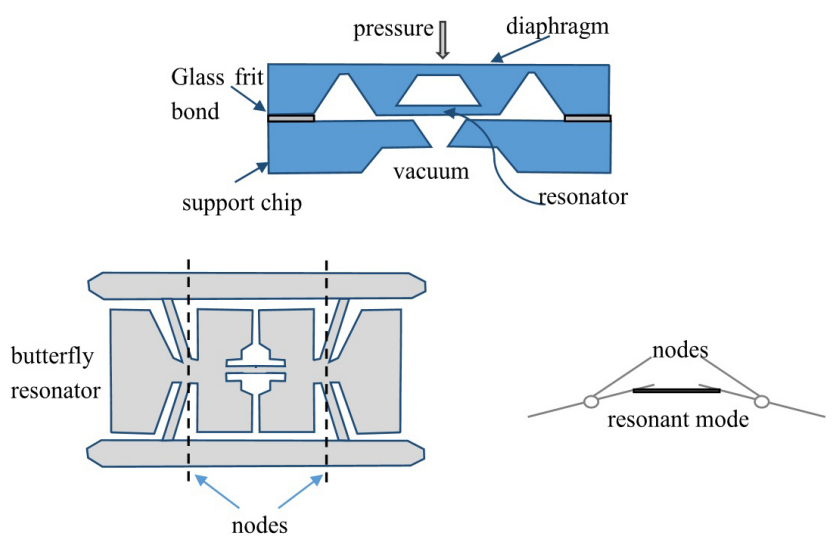

Figure 6. Druck resonant pressure sensor (Beeby et al., 2004).

\subsection{Surface acoustic wave}

Surface acoustic wave (SAW) pressure sensors are well suited for automotive application because they are designed to be passive, rugged, and extremely small. SAW sensors are different in their sensing mechanisms compared to other resonant sensors in which a mechanical resonating structure is employed. In SAW sensors, a piezoelectric crystal is used as the resonating element. This element resonates acoustic wave resonance by applying an oscillating voltage through the electrodes due to the inverse piezoelectric effect (Cavalloni et al., 2002).

SAW technology was originally used in electronic analogue signal processing in the 1970s as filters in the field of radio transmission in wireless systems (Yurish and Gomes, 2005). However, SAW sensor structure is sensitive to some physical quantities like strain, temperature, humidity, acceleration, etc., and therefore can be applied to measure these quantities (Reindl et al., 1996; Kalinin, 2004; Chin et al., 2010; Hew et al., 2011). Figure 7 shows different strain measurement mechanisms that can be employed when using SAW sensors.

A SAW measurement system is capable of wirelessly monitoring applications and acts as a passive sensor in such a way that the oscillation frequency of the transmission signal passing through the SAW sensor changes in speed or phase with the measured value. Consequently, applying SAW sensors in a tyre pressure and temperature monitoring system has been explored for their advantages in the sense that they have very small size and weight, can pass signals wirelessly, receive the required power wirelessly by the energy of the RF field (and therefore no local battery is needed), and can resist harsh environment conditions (Pohl et al., 1997, 1998; Xiangwen et al., 2004; Zhang et al., 2004; Li et al., 2010). The working principle of SAW sensors is explained in detail in chapter five in Yurish and Gomes (2005). One of the problems associated with a SAW sensing system is its short transmission range; therefore, the transmitting and receiving antennas of the read-out system have to be mounted close to
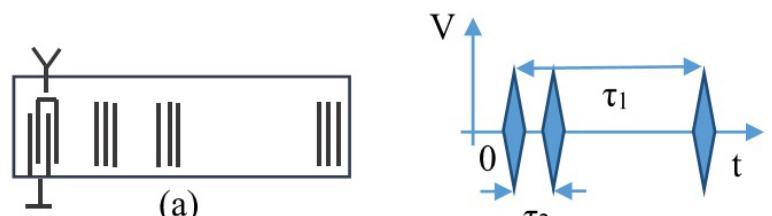

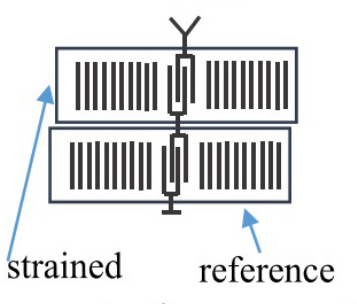

(b) resonator $\mathrm{f}_{2}$ resonator $\mathrm{f}_{1}$

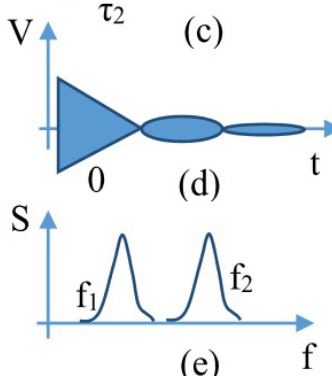

(e)
Figure 7. Reflective delay line sensor (a), resonant sensor (b), impulse responses of the delay line (c), the resonant (d) sensors, and the spectrum of the resonator response (e) (Kalinin 2004).

the SAW sensors, with a maximum distance for detection of $40 \mathrm{~cm}$ (Oh et al., 2008). In the TPMS application, for example, cables from the central read-out circuit need to be installed with antennas in their ends nearby each tyre, which increases the overall weight and the installation cost of the system. Although SAW sensors do not need batteries, the system, particularly the read-out part, is not self powered and therefore consumes power from the vehicle battery.

Transense, a company based in the UK, has developed a SAW-based tyre pressure monitoring system (Stack TPMS) which is supposed to be available on the market for motorsport applications ${ }^{3}$. SAW sensors in this system are attached to the tyre's valve and communicate with the read-out circuit antenna at $433 \mathrm{MHz}$ via a $5 \mathrm{~mm}$ diameter antenna with an optional length to be specified by the customer. Transense claims that their SAW tyre pressure sensor has $1 \mathrm{~m}$ of reading range. The system performance and characteristics are clearly explained by Dixon et al. (2006).

SAW transponders can also be designed in such a way that an external sensor, e.g. a capacitive sensor, can be connected to a reflector within the SAW chip and act as an impedance load on that reflector. Schimetta et al. (2000) present a SAW hybrid tyre pressure sensor in which a capacitive pressure sensor is used for pressure measurement. In this system, the reflectivity of the impedance loaded reflector will be a function of the variable sensor impedance, which is a function of the measured value, e.g. pressure. Such a system has the advantage of a high signal-to-noise ratio and a larger modulation factor compared to ordinary SAW sensors. However, this type of sensor is more complex to model and detect. The

\footnotetext{
${ }^{3}$ Transense, available at: http://www.transense.co. uk/technologies/temppressure/motorsport-tpms and http: //www.stackltd.com/tpms.html, last access: 16 December 2011.
} 


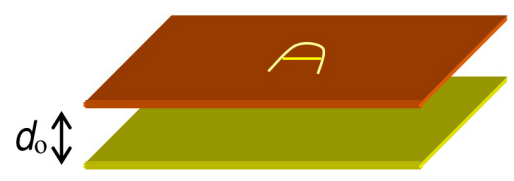

Figure 8. Schematic of a general capacitive sensor.

principle and design of a TPMS based on a hybrid SAW sensor are presented in Schimetta et al. (2000).

In a previous publication (Kubba and Jiang, 2014), the author made a comparison between the above-listed pressure sensing mechanisms and a justification was made for choosing the capacitance sensing technique. Therefore, the following sections focus on capacitive pressure sensors.

\section{Transduction mechanism of capacitive pressure sensor}

In this section the capacitive transduction is explained in detail. Traditionally, a basic capacitor consists of two parallel plates, or electrodes, of equal area $A$ and at a distance of $d_{o}$ apart (Fig. 8), and a dielectric material in-between. The mathematical expression of the electrical capacitance of the two plates can be obtained as follows (Beeby et al., 2004):

$C=\varepsilon_{o} \varepsilon_{\mathrm{r}} \frac{A}{d_{o}}$,

where $\varepsilon_{o}$ is the free space permittivity and equals $8.854 \times 10^{-12}$ in Farad per metre $\left(\mathrm{F} \mathrm{m}^{-1}\right), \varepsilon_{\mathrm{r}}$ is the relative permittivity of the dielectric material in between the capacitor electrodes, $A$ is the area of overlap between the electrodes in metres squared $\left(\mathrm{m}^{2}\right)$, and $d_{o}$ is the separation between the electrodes in metres $(\mathrm{m})$.

In order to achieve capacitive transduction, one of the independent variables in Eq. (1) has to vary with a measured quantity. This can be done either by moving the dielectric material or by moving the electrodes linearly with respect to each other. The latter is quite common in capacitive sensors due to its simplicity.

A diaphragm-based capacitive sensor has one of the electrodes acting as a diaphragm that is sensitive to pressure, resulting in a change in the height distribution between the electrodes when pressure is applied, and therefore change in the capacitance of the sensor can be detected. The usual way that capacitive absolute pressure sensors are designed is illustrated in Fig. 9. The lower plate of the capacitive sensor is rigid and stationary, while the upper one is flexible and pressure sensitive. When pressure is applied, the upper plate deforms and the average distance to the stationary plate decreases, causing an increase in capacitance. The space between the two plates is in a vacuum. A schematic diagram of the proposed capacitive pressure sensor is shown in Fig. 10. In this design, the lower and upper plates are stationary. The vacuum cavity is underneath the deformable diaphragm and

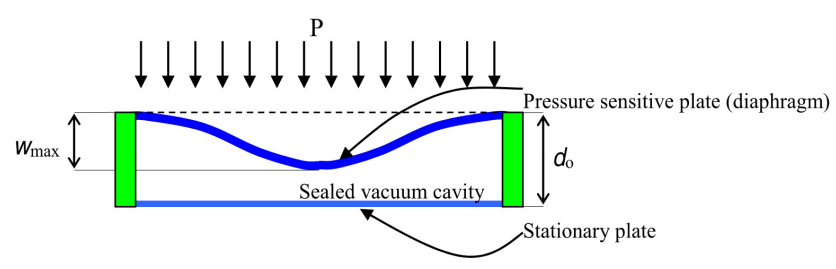

Figure 9. Schematic diagram of a general capacitive pressure sensor.

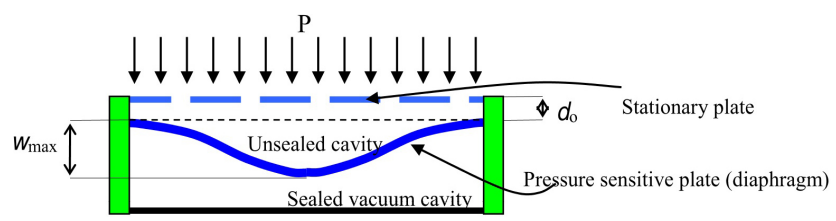

Figure 10. Schematic diagram of the proposed capacitive pressure sensor.

the upper plate is not sealed, allowing air pressure to transfer to the diaphragm.

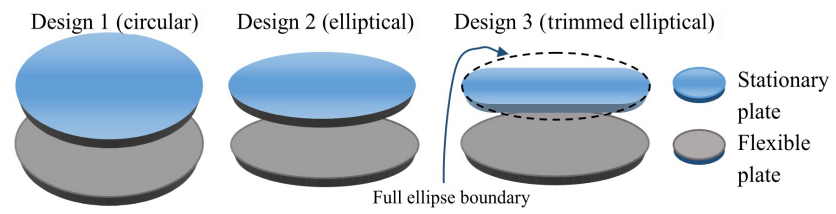

Figure 11. Capacitive sensor's functional plates of the three designs.

Unlike the conventional capacitive pressure sensors, the proposed design operates in such a way that the average distance between the capacitor plates increases when pressure is applied to the deformable diaphragm. The main advantage of this design is the avoidance of having one of the sensor electrodes within the sealed vacuum cavity and to avert the need to transfer the output signal from the inside of the sealed cavity, which can increase the long term leaking rate of the vacuum cavity. However, in the proposed design the sensor sensitivity decreases slightly with the increase in applied pressure, while conventional capacitive pressure sensors operate in the opposite way. For the application of this pressure sensor, higher sensitivity at low pressure is desirable, as in most cases detecting low tyre inflation pressure is targeted.

The following sections analyse the proposed micropressure sensor in both mechanical and electrical aspects.

\section{Sensor geometry}

This section examines three possible designs of the diaphragm-based micro-pressure sensor shown in Fig. 10. It is assumed that sensors in these designs have the same pressure sensitive diaphragm area in either circular or elliptical geometries as shown in Fig. 11. The first two designs have 
their upper capacitor electrode fully covering the lower pressure sensitive electrode, while the third has the upper plate partially covering the lower capacitor plate. The upper plate in design 3 has two segments of chords parallel to the major axis removed, that is, the area between chords and dashed lines in design 3 in Fig. 11, making up $70 \%$ of the full ellipse upper plate area in design 2 . The aspect ratio (major to minor axis ratio) of the elliptical diaphragm in the second and third designs is 2 . These designs are then compared in terms of pressure sensitivity, temperature dependence, and stress levels.

In the literature, comparisons between different basic diaphragm geometries with equal areas are presented in Wang and Ko (1999) and Muhammad (2012). The comparisons show that a circular diaphragm has the lowest maximum stress and the highest sensitivity among the basic shapes, that is, square, rectangular and circular diaphragms with constant area. Elliptical diaphragms that have the same area as a circular diaphragm have higher stiffness, and therefore cover a higher pressure detection range. In addition, having higher stiffness means higher dynamic stability when included within a dynamic domain (Swett, 2012). In this section, a comparison between a circular and elliptical diaphragm is made in order to determine which is more suited for the TPMS application.

\section{Sensor analysis}

\subsection{Analytical mechanical analysis}

This section focuses on the analytical calculations of the deformable diaphragm within the micro-capacitive pressure sensor for both circular and elliptical diaphragms. The obtained analytical diaphragm deformation expressions, for both the circular and elliptical geometries, can be used to determine the enclosed capacitance between the deformable diaphragm and a stationary plate located on top of it, separated by distance $d_{o}$ as illustrated in Figs. 10 and 11 .

Despite the lack of accuracy associated with most analytical calculations usually resulting from assumptions and approximations, analytical calculations can still offer a rather nimble and useful way of gaining a valuable answer, or of comprehending the physical process behind a particular problem. In the case of designing a pressure sensitive diaphragm, analytical techniques can be used to estimate the deflection response when subjected to uniform pressure. The analytical formulae applied in this section are quoted from "Theory of plates and shells" by Timoshenko (1940). These formulae are based on the following assumptions (Beeby et al., 2004).

- The diaphragm is not curved when unloaded and has a uniform thickness.

- The diaphragm substance is homogenous and isotropic.
- The pressure on the diaphragm is uniformly distributed and perpendicular to the diaphragm plan.

- The applied pressure is within the elastic loading limits and does not cause any plastic deformation.

- The diaphragm thickness to diameter ratio is less than $20 \%$.

- All deformation occurring in the diaphragm is due to bending only, and the neutral axis of the diaphragm stays at a zero stress level.

The mathematical expression of the deflection of a circular and elliptical plates under uniform pressure with clamped edges can be obtained using the expression given in Timoshenko (1940).

As mentioned earlier, and in order to compare between circular and elliptical diaphragms, thickness and surface area in both geometries are identical.

Hence

$A_{o}=A_{0}$,

where $A_{o}$ is the circular diaphragm area in $\mathrm{m}^{2}$ and $A_{0}$ the elliptical diaphragm area in $\mathrm{m}^{2}$. Then

$\pi a_{o}^{2}=\pi a b$.

As such, the radii of both geometries can be expressed as

$a_{o}=\sqrt{a b}$,

where $a_{o}$ is the diaphragm outer radius in $\mathrm{m}$, and $a$ and $b$ are major and minor axes respectively, both in $\mathrm{m}$.

If

$a=2 b$,

Eq. (4) can be re-written as

$a=\sqrt{2 a_{o}}$.

The proposed design uses Eq. (6) to relate between the circular and elliptical diaphragm diameters.

In terms of the stresses acting on the diaphragm edge as a result of the diaphragm deflection, the elliptical geometry offers a lower stress level near the major axis edge compared to the circular diaphragm geometry (Timoshenko, 1940). This fact can be exploited when bonding the capacitor terminals; that is, the bonding and isolating substance (SU-8) can be developed over that low stress area. Feng and Farris (2003) show that the process of fabricating SU-8 can change its mechanical properties. In this research, it is assumed that the SU-8 mechanical properties and shear bonding strength are as presented by Pang et al. (2008) and Guerin (2008) respectively. This technique improves the overall sensor robustness and reduces the temperature sensitivity of the transducer. As 
Table 1. Circular and elliptical diaphragms' design parameters.

\begin{tabular}{|c|c|}
\hline Parameter & Value \\
\hline$a_{o}$ & $1.25 \mathrm{~mm}$ \\
\hline$a$ & $1.767767 \mathrm{~mm}$ \\
\hline$b$ & $0.883883 \mathrm{~mm}$ \\
\hline$h$ & $100 \mu \mathrm{m}$ \\
\hline$E_{\text {Si[plate bending] }}{ }^{*}$ & $170 \mathrm{GPa}$ \\
\hline$E_{\mathrm{SU}-8}$ & $4.4 \mathrm{GPa}$ \\
\hline$v_{\text {Si[plate bending }]}{ }^{*}$ & 0.064 \\
\hline$v_{\mathrm{SU}-8}$ & 0.22 \\
\hline$\alpha_{\mathrm{Si}}$ & $2.6\left(\mathrm{ppm}^{\circ} \mathrm{C}^{-1}\right)$ \\
\hline$\alpha_{\mathrm{SU}-8}$ & $52\left(\mathrm{ppm}^{\circ} \mathrm{C}^{-1}\right)$ \\
\hline$\left(\varepsilon_{\mathrm{r}}\right)_{\mathrm{SU}-8}$ & 3 \\
\hline
\end{tabular}

these stresses are rather difficult to calculate manually, particularly the calculations of the thermal stresses' effect, numerical simulations with COMSOL software were used. The numerical results are presented in Sect. 4.3.

The dimensions and bi-dimensional silicon elastic properties for plate bending (Hopcroft et al., 2010) of the circular and elliptical diaphragms are shown in Table 1 . The radii of the elliptical diaphragm are calculated using Eqs. (5) and (6), where $\alpha_{\mathrm{Si}}$ and $\alpha_{\mathrm{SU}-8}$ are the silicon and SU- 8 coefficients of thermal expansion (CTEs) respectively. Provided that silicon is an anisotropic material, and that a silicon wafer orientation of (100) is the most common case of MEMS fabrication, silicon mechanical properties presented in Hopcroft et al. (2010), which are also proven experimentally by Boyd and Uttamchandani (2012), were applied in COMSOL simulations. However, in the analytical calculations, values were used assuming plate bending for $\langle 110\rangle$ in (100) silicon to simplify initial design calculations.

\subsection{Analytical capacitance modelling and calculations}

By considering the sensor schematic illustrated in Fig. 10, the capacitance enclosed between the stationary plate and a clamped circular deformable diaphragm subjected to a uniformly distributed pressure can be obtained:

$C(p)=8 \pi \varepsilon_{o} \varepsilon_{\mathrm{r}} \sqrt{\frac{D}{d_{o} p}} \tan ^{-1}\left(-\frac{a_{o}^{2}}{8} \sqrt{\frac{p}{d_{o} D}}\right)$,

where $\varepsilon_{o}$ is the free space permittivity and equals $8.854 \times 10^{-12}$ in Farad per metre $\left(\mathrm{F} \mathrm{m}^{-1}\right), \varepsilon_{\mathrm{r}}$ is the relative permittivity of the dielectric material in between the capacitor electrodes, $D$ is the flexural rigidity in $N \mathrm{~m}^{2}$, and $p$ is the pressure in $\mathrm{Pa}$.

In the case of the elliptical diaphragm, the capacitance expression can be obtained by defining the diaphragm radius

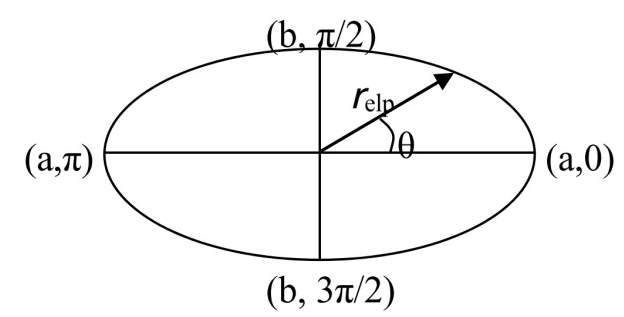

Figure 12. Ellipse border representation using the polar coordinate system.

using the polar coordinate system with the origin at the centre of the ellipse (Fig. 12), and then by integrating over the whole oval area using double integration. This results in a rather complicated mathematical expression:

$C(p)=\varepsilon_{o} \varepsilon_{\mathrm{r}} \int_{0}^{2 \pi^{r_{\mathrm{elp}}}} \int_{0} \frac{r \mathrm{~d} r \mathrm{~d} \theta}{d_{o}+w_{0}\left(1-\frac{(r \cos \theta)^{2}}{a^{2}}-\frac{(r \sin \theta)^{2}}{b^{2}}\right)^{2}}$,

where $r$ and $\theta$ are the polar coordinates at which diaphragm deflection is calculated in $\mathrm{m}$ and rad respectively, $r_{\mathrm{elp}}$ is the ellipse radius at a certain angular position $\theta$ in $\mathrm{m}$, and $w_{0}$ is the central deflection of the diaphragm.

Wolfram Mathematics software ${ }^{4}$ was used to solve the first integral of Eq. (8), and it can be seen that a numerical integration could be a better choice to achieve a useful result (see Appendix A). Attempts to solve Eq. (8) with MATLAB tools were not successful for the whole pressure range, and therefore FEA tools (COMSOL) were used to evaluate the integral over a range of pressures. In order to find out COMSOL's accuracy, the circular diaphragm results from both analytical and FEA analyses are compared.

Due to the symmetrical nature of the three designs studied in this research, COMSOL simulation was undertaken for a quarter of the sensors' geometries to accelerate simulation time and to improve simulation accuracy. Threedimensional views of a quarter of the three diaphragm designs' capacitive pressure sensor configurations are shown in Figs. 13, 14, and 15 respectively.

In order to evaluate the total capacitance of the sensor shown in Fig. 13, the parasitic capacitance has to be calculated, that is, the capacitance enclosed between the upper plate and the sealing plate excluding the deformable diaphragm. Details of the analytical calculations of the parasitic capacitance, $19.59 \mathrm{pF}$, are presented in Appendix A. The elliptical diaphragm design has the same area as the circular design but with an aspect ratio of $2: 1$ (Fig. 14); therefore, both designs share the same parasitic capacitance value. The capacitance-pressure curve of the circular diaphragm pressure sensor presented in Fig. 13 is shown in Fig. 16. These data were calculated both analytically using MATLAB and

\footnotetext{
${ }^{4}$ http://integrals.wolfram.com
} 


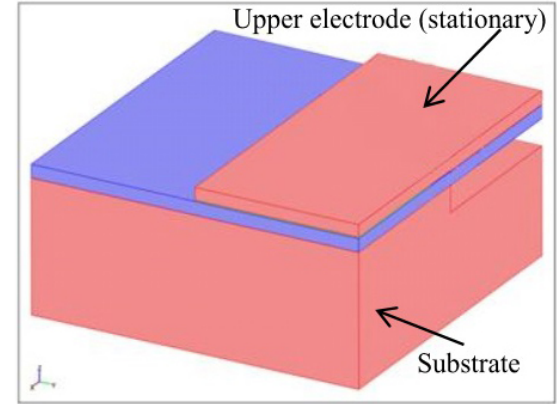

Isometric view

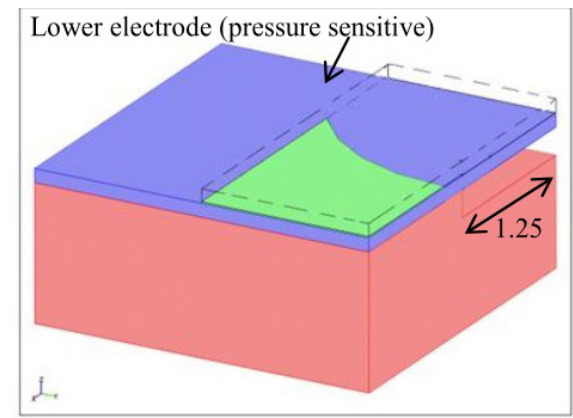

Isometric view with no upper electrode

Top view with no upper electrode

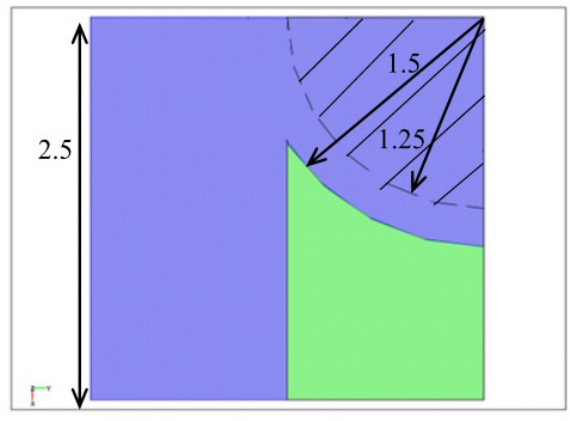

\begin{tabular}{|l|}
\hline Design 1 \\
$\square$ SU-8 \\
$\square$ Lower electrode's \\
pressure sensitive \\
area \\
$\square$ Substrate \& upper \\
electrode \\
\hline
\end{tabular}

All dimensions are in $\mathrm{mm}$.

Figure 13. A three-dimensional view of a quarter of the circular diaphragm pressure sensor.

numerically using COMSOL - to be illustrated in the following section. Having the same area in both the elliptical and the circular diaphragm pressure sensor designs, the initial $0 \mathrm{~Pa}$ - capacitance values are identical and equal to $41.32 \mathrm{pF}$. In the elliptical diaphragm design the MATLAB code only converges at $1.5 \mathrm{MPa}$ applied pressure and results in a capacitance value of $36.63 \mathrm{pF}$. This shows that the elliptical diaphragm design has less change in the capacitance value within the same pressure range, that is, less sensitivity and high sensor bias to the output signal range ratio. To increase the sensor's output signal range to bias ratio, the area of the sensor upper electrode can be reduced. That is the case in the third design, shown in Fig. 15, where only $70 \%$ of the elliptical diaphragm is the functional capacitor area. Thermal response and stress levels in all the designs have to be investigated. This can be a rather complicated task for manual calculation, and therefore numerical analysis is employed as the second best alternative. The following section presents numerically simulated results, using COMSOL software, of the mechanical and electrical variables for all three designs' parameters.

\subsection{Numerical analysis}

In this section, initially, the first design (Fig. 13) simulation results are compared with the analytical results to check the reliability of the FEA results. Then, the second and third designs are simulated using FEA, and all three designs are compared in order to select the most suitable one for the TPMS application. Having the three designs symmetrically around $x-z$ and $y-z$ planes, there are two main quantities between the first design simulation results and the analytical results: the parasitic capacitance and the diaphragm deflection.

The parasitic capacitance was found analytically to be equal to 19.59 and $17.46 \mathrm{pF}$ using FEA. This is due to the approximation made by the software by replacing arcs with sets of straight lines giving slightly smaller areas for both the diaphragm and SU-8 layer. This difference can be treated as bias, or offset, error, and therefore it will not affect the sensor sensitivity calculations.

Regarding the diaphragm deflection, it is worth mentioning that the analytical diaphragm deformation analysis for the first design is based on a clamped-edge mounting condition, which might not be the exact case for how the diaphragm is fixed onto the sensor substrate. Having the diaphragm bonded from one side, its bending stiffness under uniform pressure is less than the clamped-edge mounting stiffness. For this reason, FEA results might give a higher diaphragm deflection under a certain pressure than that obtained using the analytical analysis described in Sect. 4.1 (see Fig. 16). It can be seen that a slightly higher central deflection $\left(w_{o}\right)$ is obtained compared to the calculated one using clamped-edge mounting, in which higher flexural rigidity exists.

The temperature sensitivity of the sensor-resulting capacitance for the three designs is shown in Fig. 19.

The reasonable agreement between the analytical results and the numerical results obtained for design 1 supports the use of FEA simulations for designs 2 and 3. Diaphragm de- 


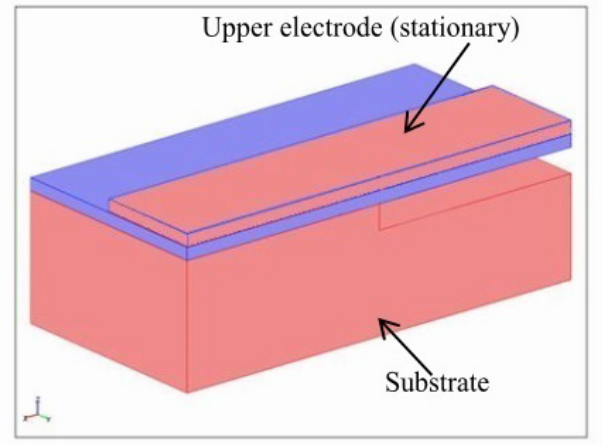

Isometric view

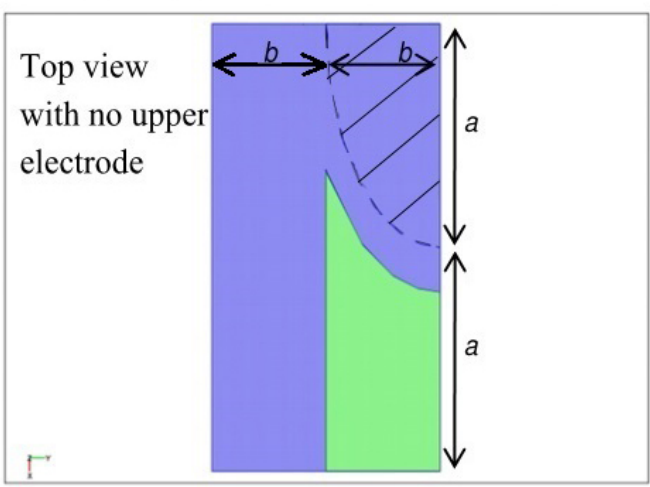

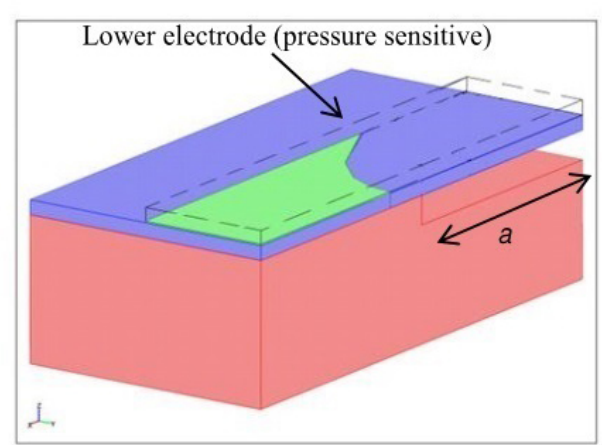

Isometric view with no upper electrode

\begin{tabular}{|l|}
\hline Design 2 \\
$\square$ SU-8 \\
$\square$ Lower electrode's \\
pressure sensitive \\
area \\
$\square$ Substrate \& upper \\
electrode
\end{tabular}

Figure 14. A three-dimensional view of a quarter of the elliptical diaphragm pressure sensor.

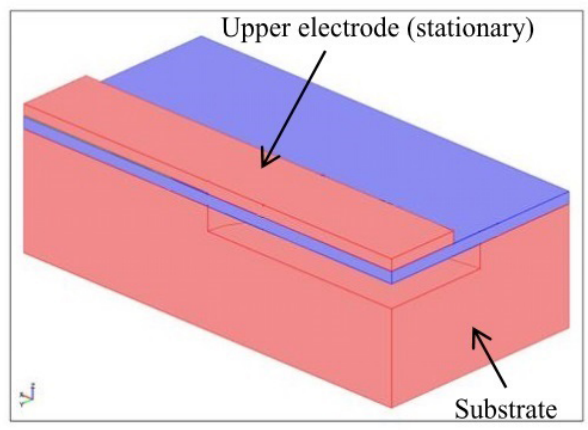

Isometric view

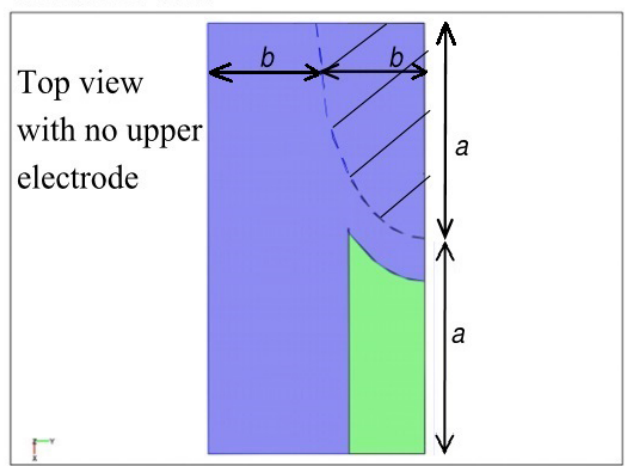

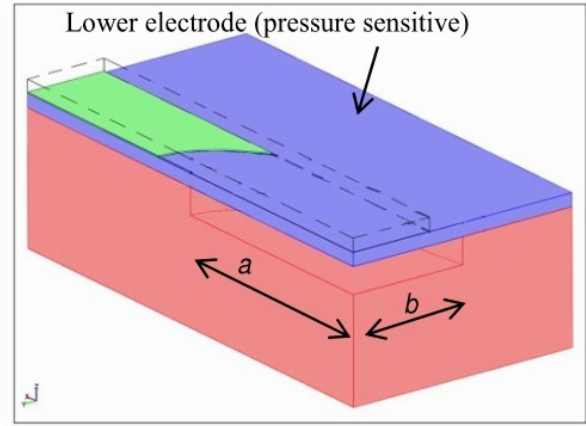

Isometric view with no upper electrode

Figure 15. A three-dimensional view of a quarter of the narrowed elliptical diaphragm pressure sensor. 


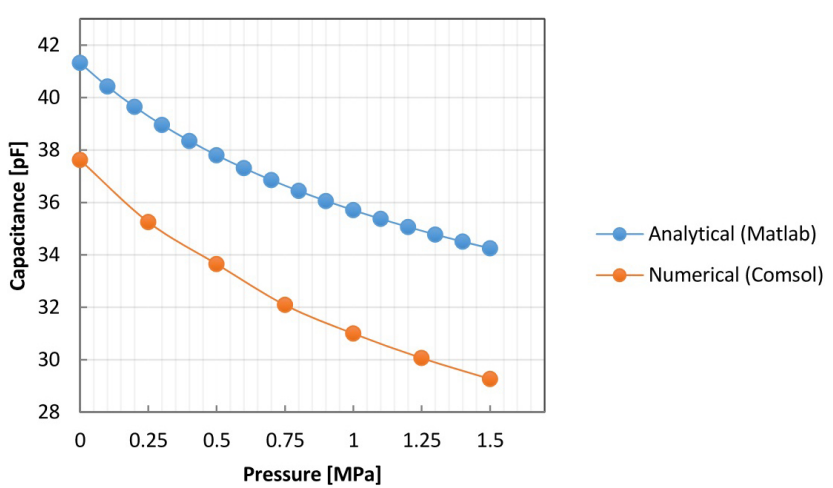

Figure 16. Capacitance-pressure curve of the circular diaphragm in design 1 using FEA.

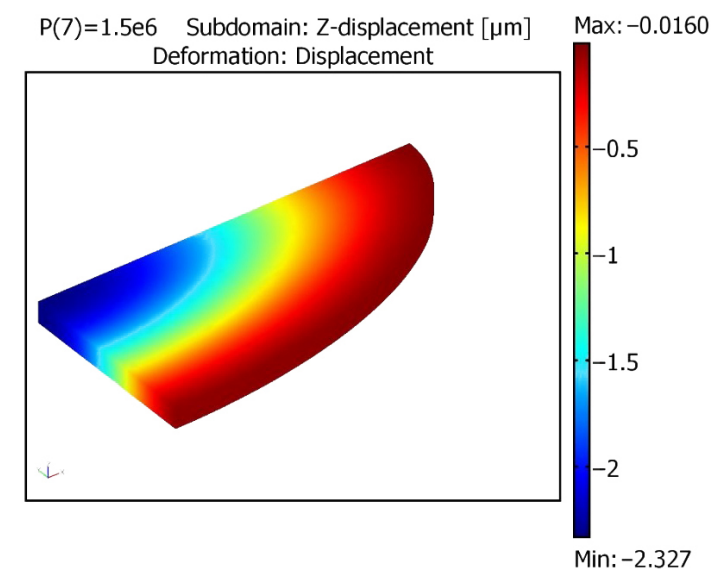

Figure 17. A quarter of the elliptical diaphragm displacement in design 2 using FEA.

formation and the sensor output signal at 20 and $100^{\circ} \mathrm{C}$ for designs 1 and 2 are shown in Figs. 17-26. The misalignment between the analytical and numerical results for design 1 is attributed to the assumption of isotropic behaviour in the diaphragm deflection calculations, whereas COMSOL applies the actual anisotropic mechanical properties of a (100) plane oriented silicon wafer as illustrated in Hopcroft et al. (2010).

By comparing the output signal of design 2 with design 3, it can be seen that design 3 offers lower bias and lower temperature sensitivity on the output capacitance. This is attributed to the smaller parasitic capacitance caused by the SU-8 layer. In addition, the simulation results listed in Table 2 show that design 3 has the lowest stress levels among all three designs, particularly the bonding shear stress between Si and SU-8, which should not exceed 17.15 MPa (Pang et al., 2008). Bonding surface shear stress in the presented designs results from the difference in thermal expansion between SU-8 and $\mathrm{Si}$. It is determined within the surface using COMSOL after increasing the temperature by $80^{\circ} \mathrm{C}$.

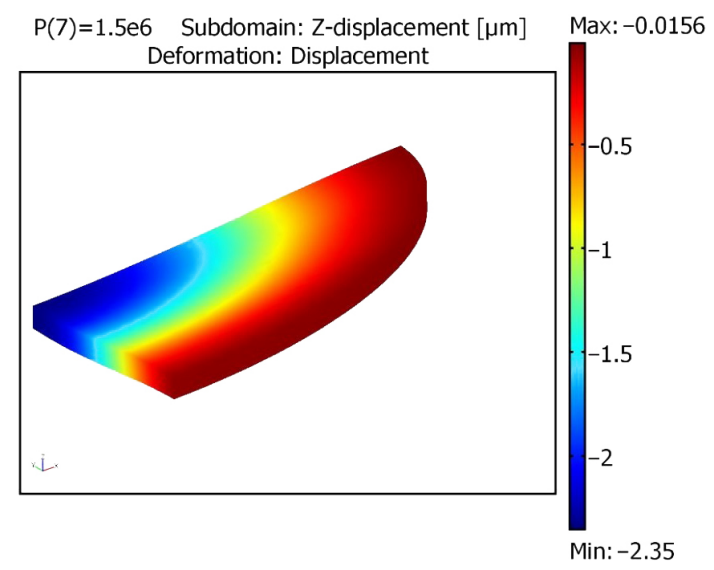

Figure 18. A quarter of the elliptical diaphragm displacement in design 3 using FEA.

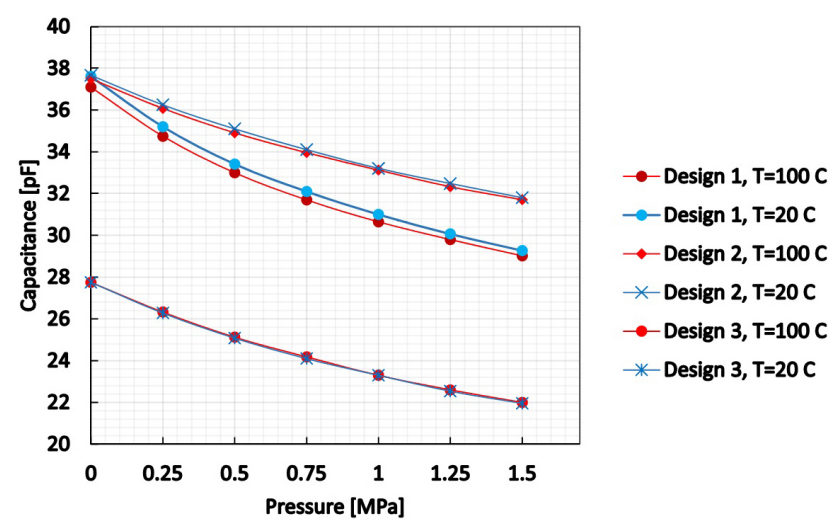

Figure 19. Temperature effect on the output signal in design 3 using FEA.

\section{MEMS capacitive pressure sensor micro-fabrication}

This section presents suggested micro-fabrication processes for making the designed MEMS pressure sensor, that is, design 3. However, due to the limited budget of this research project and the lack of essential micro-fabrication equipment needed for this design, there were no micro-fabrication processes involved in the study. Therefore, the following process flow gives only the general, and not detailed, microfabrication steps involved in making the designed micropressure sensor, as it is not the objective of this research.

Silicon is a dominant material in the semi-inductor industry due to its availability, low costs, suitability to the requirements of a wide range of mechanical and electrical properties, readiness of mature batch fabrication techniques, and the potential for integration with electronic circuitry (Petersen, 1982; Beeby et al., 2004; Hsu, 2008). For these reasons, silicon is employed in the proposed micro-pressure sensor in the diaphragm, the conductive plates (electrodes) of the capacitor, and the vacuum chamber of the sensor. 
Table 2. A summarized comparison of the three capacitive pressure sensor designs.

\begin{tabular}{|c|c|c|c|c|c|c|c|}
\hline \multirow{2}{*}{\multicolumn{2}{|c|}{$\frac{\text { Item }}{\text { Property }}$}} & \multicolumn{2}{|c|}{ Design 1} & \multicolumn{2}{|c|}{ Design 2} & \multicolumn{2}{|c|}{ Design 3} \\
\hline & & $T=20^{\circ} \mathrm{C}$ & $T=100^{\circ} \mathrm{C}$ & $T=20^{\circ} \mathrm{C}$ & $T=100^{\circ} \mathrm{C}$ & $T=20^{\circ} \mathrm{C}$ & $T=100^{\circ} \mathrm{C}$ \\
\hline \multirow{2}{*}{ 文 } & $\begin{array}{c}\sigma_{\max }(\text { Von Mises }) \\
(\mathrm{MPa})\end{array}$ & 1.48 & 58 & 2.4 & 44.44 & 0.12 & 40.17 \\
\hline & $\begin{array}{c}\text { Bonding surface } \\
\tau_{\max }(\mathrm{MPa})\end{array}$ & 0.05 & 9.85 & 0.11 & 2.35 & 0.002 & 0.827 \\
\hline $\bar{\sim}$ & $\begin{array}{c}\sigma_{\max }(\text { Von Mises }) \\
(\mathrm{MPa})\end{array}$ & 171.17 & 191 & 111 & 120.23 & 113.58 & 119.86 \\
\hline \multicolumn{2}{|c|}{$\begin{array}{l}\text { Average sensitivity } \\
\qquad\left(\mathrm{pF} \mathrm{bar}^{-1}\right)^{\mathrm{a}}\end{array}$} & \multicolumn{2}{|c|}{0.558} & \multicolumn{2}{|c|}{0.39} & \multicolumn{2}{|c|}{0.386} \\
\hline & $\Delta C / C$ & \multicolumn{2}{|c|}{-0.22} & \multicolumn{2}{|c|}{-0.155} & \multicolumn{2}{|c|}{-0.208} \\
\hline
\end{tabular}

a Sensitivity was averaged by considering the slope of the best fitting straight line, i.e. by linear regression.

The photolithography processes required in the proposed design are mainly etching the vacuum cavity in the lower part of the sensor structure and etching the upper stationary plate in order to allow air pressure to pass to the pressure sensitive diaphragm and to determine the overlapping surface area between the two capacitor plates.

In order to construct an absolute capacitive pressure sensor, bonding between the sensor layers is essential. The vacuum chamber has to be sealed hermitically and be able to withstand temperature variation. This can be done by first sealing the vacuum chamber and then isolating the capacitive electrodes. From the literature, one reliable bonding technique is anodic bonding, which is the most suited bonding method for sealing the vacuum chamber in the proposed micro-pressure sensor. Silicon-to-silicon anodic bonding can be obtained by applying sputtered borosilicate glass as a thin film layer on either of the silicon surfaces to be bonded, as demonstrated in Chapter 9 in Halbo and Ohlckers (1995). In the proposed design, a cross section is shown in Fig. 20, the vacuum chamber is sealed hermetically using anodic bonding, and the electrodes are bonded, and isolated, using SU-8 photoresist. SU-8 is low cost, easy to process, and a good isolation material for capacitive pressure sensor application, and it can work as an adhesive bonder too (Chang and Allen, 2004; Pang et al., 2008).

The main micro-fabrication steps include wet etching for $\mathrm{Si}$, Si to Si anodic bonding, and $\mathrm{Si}$ to $\mathrm{Si}$ adhesive bonding using SU-8 (see Fig. 20).

\section{Sensor read-out circuitry}

This section presents a read-out and low power short-range transmission circuit suited to capacitive sensors. This circuit is based on a negative resistance lambda diode RF oscillator which contains a tuned $L C$ circuit for both measurement and transmission purposes.
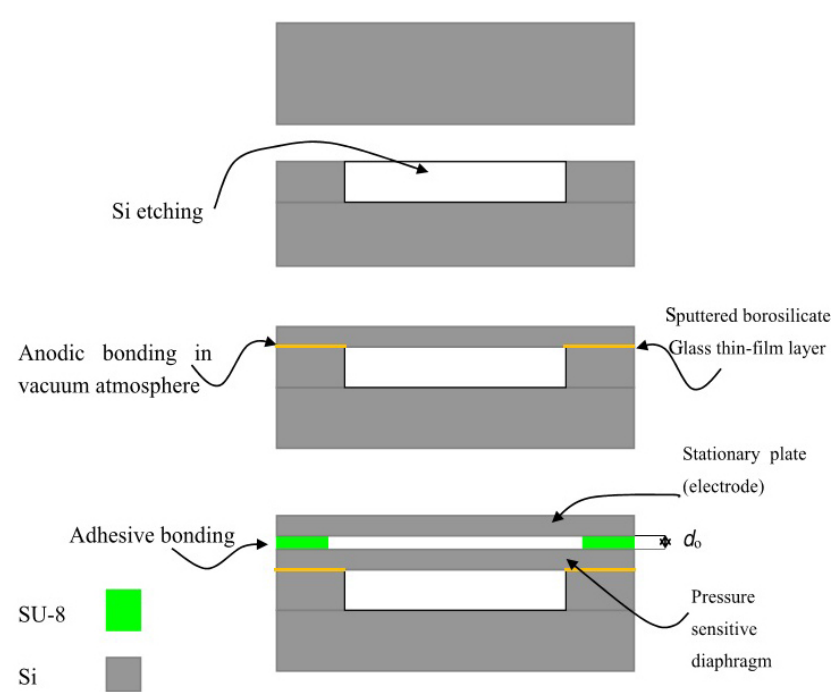

Figure 20. A suggested micro-machining process flow of the designed MEMS pressure sensor.

In the literature, a tunnel diode oscillator was employed by Suster et al. (2002) for a high-temperature wireless pressure sensor node. The simplicity of this system offers very low transmission power $(<1 \mathrm{~mW})$, which is very attractive for energy harvesting systems. Suster et al. (2003) demonstrated an optically powered wireless pressure sensor node based on a tunnel diode oscillator using a photo-diode as the energy harvesting unit. As tunnel diodes were not commercially available, equivalent combinations of JFET transistors acting as a negative resistance, so-called lambda diodes, were employed. When a pair of JFETs ( $N$-channel and $P$-channel) is connected in a certain way, they can serve as a negative resistance within a negative resistance oscillator (Chua et al., 1985). A schematic diagram of the lambda diode oscillator is shown in Fig. 21a. In this circuit, the $L C$ circuit oscillates at 


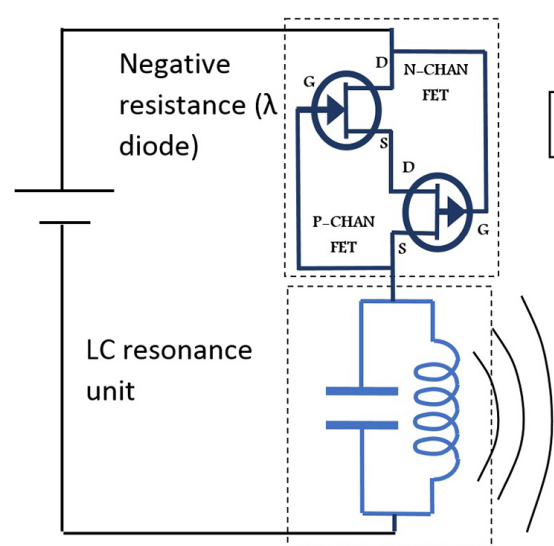

(a)

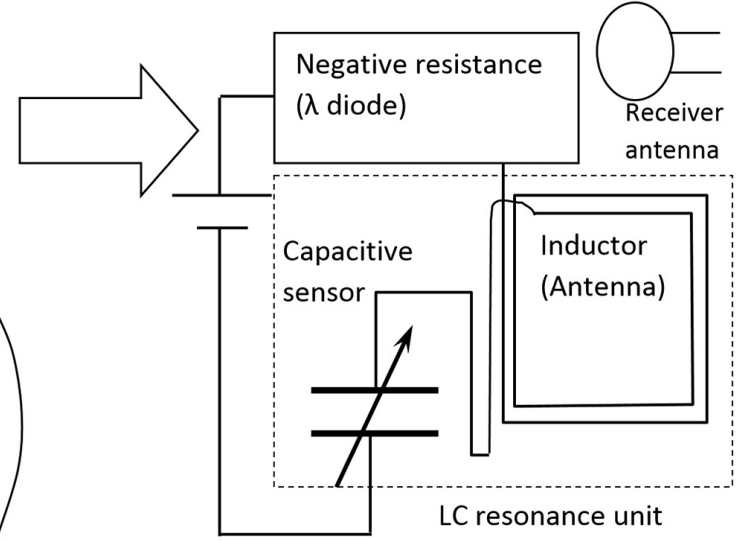

(b)

Figure 21. (a) Negative resistance oscillator schematic; (b) capacitive sensor read-out and transmission circuit. Electronic circuit design published by Butler (1997).

2N 3819-20 JFETs pair

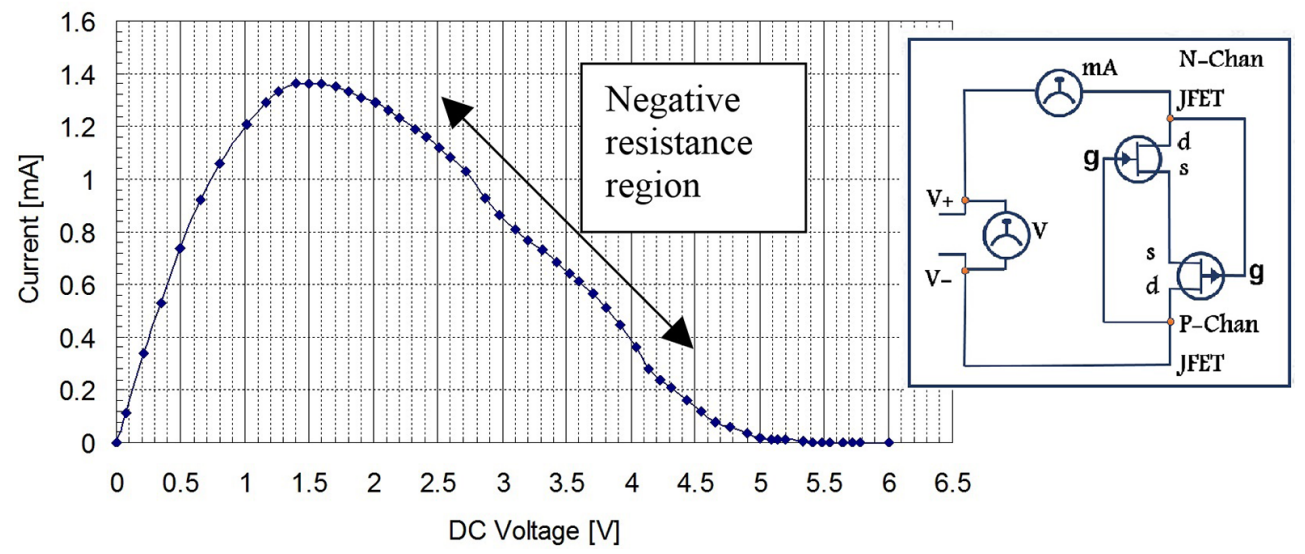

Figure 22. The 2N3819 and 2N3820 JFET pair lambda diode characteristic. Electronic circuit design published by Butler (1997).

2N5462(P-JFET),BF244B(N-JFET) pair

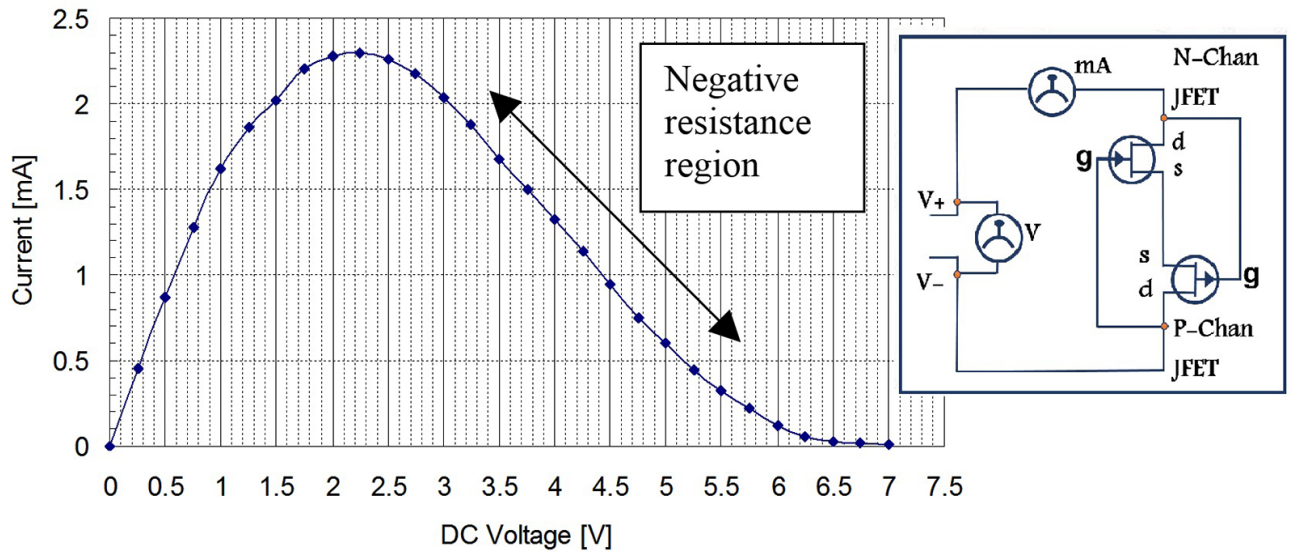

Figure 23. The 2N5462 and BF244B JFET pair lambda diode characteristic. Electronic circuit design published by Butler (1997). 


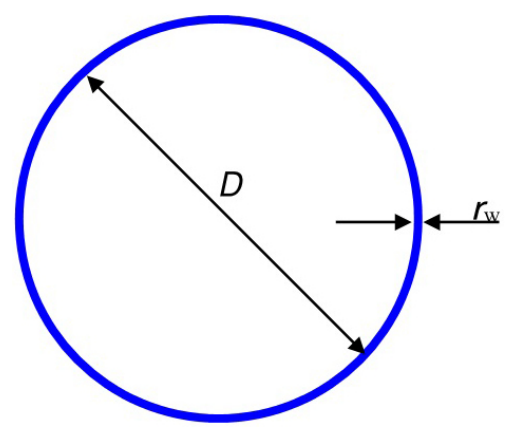

Figure 24. Loop antenna schematic.

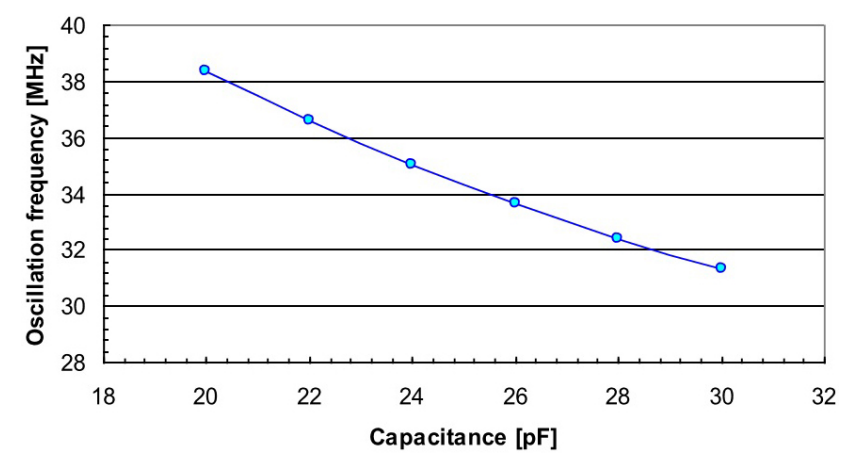

Figure 25. Predicted first harmonic oscillation frequency of the lambda diode oscillator when its tuning circuit contains the design 3 capacitive pressure sensor and the loop antenna of Fig. 24.

resonance frequency and its harmonics (multiples), as shown in the equation below:

$f=n \frac{1}{2 \pi \sqrt{L C}} ; n=1,2,3, \ldots \infty$

When the applied voltage is within the negative resistance regain of the contained lambda diode, which is in parallel with the $L C$ circuit, oscillation frequency might not however follow Eq. (9) exactly. Two JFETs pairs were tested experimentally and their voltage-current characteristics are shown in Figs. 22 and 23.

A variable capacitor may act as the capacitor in the $L C$ circuit as shown in Fig. 21b; the tuning frequency will vary according to the capacitance values and the capacitance value can be found by measuring the oscillator resonance frequency. The variable capacitor can be a capacitive sensor, and therefore the oscillation frequency can reflect the measured pressure value. The second part of the $L C$ tuning circuit is the inductor, which in this design acts as the antenna of the oscillator, or the transmitter, and an external receiver with an identical antenna can detect the oscillation frequency.

The inductance of a circular loop antenna contains $N$ identical loops, shown in Fig. 24, and can be approximated using
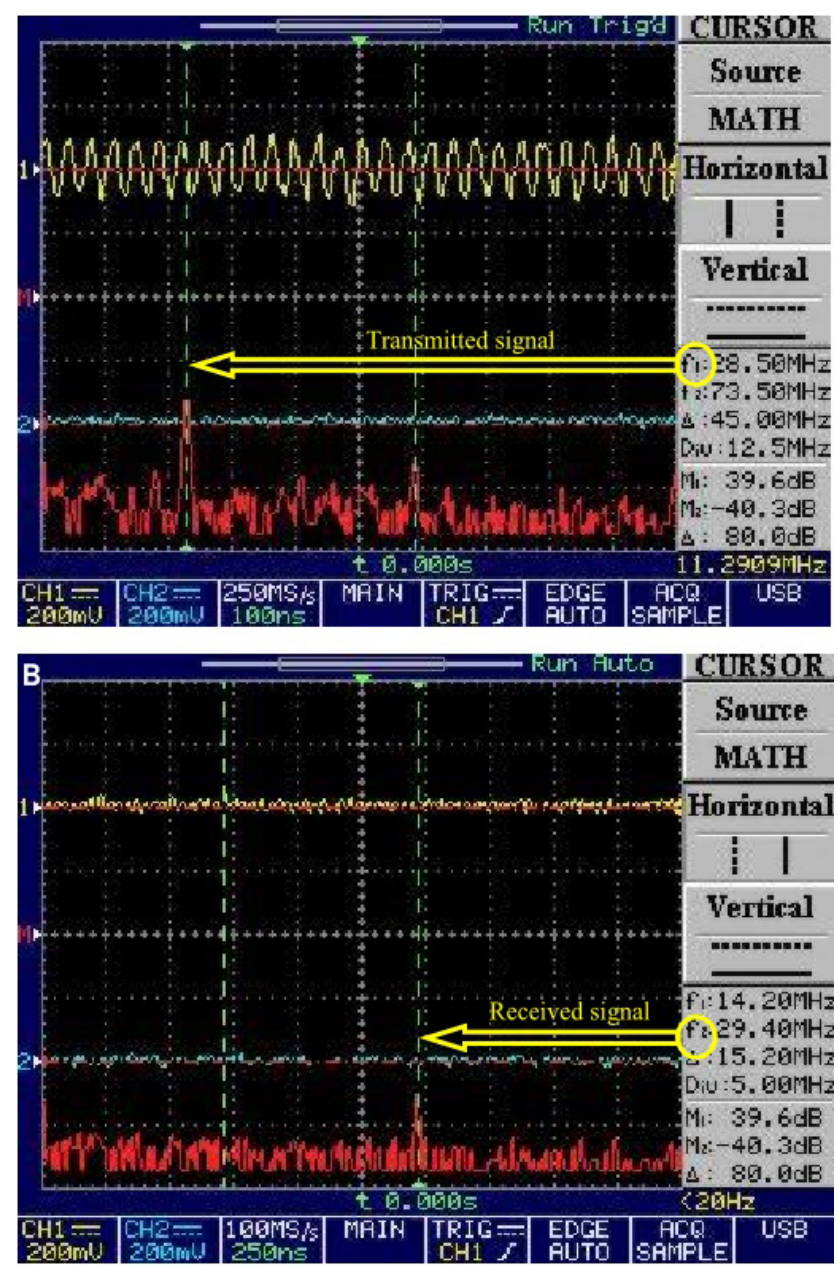

Figure 26. Lambda diode oscillator transmitted and received signals conducted using a digital storage oscilloscope: (a) transmitted signal; (b) received signal.

the following formula (Paul, 2010):

$L_{N \text { loops }} \cong N^{2} \frac{\mu_{\mathrm{r}} \mu_{0} D}{2}\left(\ln \frac{4 D}{r_{\mathrm{w}}}-2\right)$,

where $\mu_{\mathrm{r}}$ is the relative permeability, in this case essentially for air, and equals $1, \mu_{0}$ is the permeability of the free space and equals $4 \pi \times 10^{-7}\left(\mathrm{H} \mathrm{m}^{-1}\right), D$ is the loop diameter in $\mathrm{m}$, and $r_{\mathrm{w}}$ is the wire diameter in $\mathrm{m}$.

Given that $N$ equals $2, r_{\mathrm{w}}$ equals $0.6 \mathrm{~mm}$ and $D$ equals $80 \mathrm{~mm}$, using Eq. (10), the inductance of the loop antenna will be approximately $860(\mathrm{nH})$.

If the simulated capacitive pressure sensor (design 3) is substituted as the variable capacitor in the tuning circuit of the lambda diode oscillator shown in Fig. 21a, and using the estimated inductance of the loop antenna, that is, 860 $(\mathrm{nH})$, the first harmonic oscillation frequency from Eq. (9) of the oscillator will be obtained as shown in Fig. 25. This graph shows an average sensitivity of $1.4 \mathrm{MHz} \mathrm{pF}^{-1}$, meaning a sensitivity of $0.543 \mathrm{MHz}^{-1}$ for the simulated (de- 
sign 3) MEMS capacitive pressure sensor. It is worth mentioning that the parasitic capacitance of the loop antenna was neglected.

Figure 26 shows the lambda diode oscillator output signal as measured using a digital storage oscilloscope (DSO), manufactured by TENMA model no. 72-7240. The tuning circuit contains a trimmer capacitor (approximate range $10-50 \mathrm{pF}$ ) to simulate the variable capacitance of the MEMS pressure sensor and the loop antenna shown in Fig. 24. The conditions at which these results were obtained were that both the transmitting and receiving antennas were identical $(0.6 \mathrm{~mm}$ wire diameter, $80 \mathrm{~mm}$ coil diameter, two turns) and mounted coaxially and separated by $110 \mathrm{~mm}$. The receiver antenna was connected directly to the DSO probes with no amplification or filtering circuits.

Despite the approximate value of the trimmer capacitor capacitance range, it was observed that the oscillation frequency changes noticeably, approximately by $10 \mathrm{MHz}$ over the trimmer capacitor capacitance range, and can be employed for measuring the variable capacitor value and ultimately the pressure value when a capacitive pressure transducer is employed. It was also noticed that there is a frequency difference of approximately $900 \mathrm{kHz}$ between the transmitted and received signals, which can be treated as an offset in the very basic receiver unit.

\section{Conclusions}

In this article, three different micro-capacitive pressure sensors are compared and a read-out and transmission circuit for a capacitive transducer are presented.

The comparison between the three simulated diaphragmbased pressure transducers shows that although a circular shaped diaphragm has higher pressure sensitivity than an elliptical one, an elliptical shaped diaphragm can offer higher thermal stability and less bonding stresses compared to a circular diaphragm, which can be a useful property for a pressure sensor, particularly when employed in a harsh environment, e.g. tyre pressure monitoring.

The presented read-out circuit is selected for its simplicity and its extremely low power consumption is suitable as part of a wireless sensor node powered by energy harvesting.

In a separate article (Kubba and Jiang, 2013), the author developed a vibration-based energy harvester which was eventually used to power the presented lambda diode readout and transmission unit. 
Appendix A: Analytical integration formulae

The following integrations were solved with the aid of an open-access online integral solver ${ }^{5}$.

$\int \frac{e x}{d+w\left(1-\frac{(x \cos (t))^{2}}{a^{2}}-\frac{(x \sin (t))^{2}}{b^{2}}\right)^{2}} \mathrm{~d} x=\frac{\left(e a^{2} b^{2} \tan ^{-1}\left(\frac{\left(2 a^{2} b^{2} \sqrt{d}\left(\left(b^{2}-a^{2}\right) \cos 2 t+a^{2}+b^{2}\right)\right)}{\sqrt{w\left(\left(b^{2}-a^{2}\right) \cos 2 t+a^{2}+b^{2}\right)^{2}\left(x^{2}\left(a^{2}-b^{2}\right) \cos 2 t+a^{2}\left(2 b^{2}-x^{2}\right)-b^{2} x^{2}\right)}}\right)\right)}{\left(\sqrt{d} \sqrt{w\left(\left(b^{2}-a^{2}\right) \cos 2 t+a^{2}+b^{2}\right)^{2}}\right)}$

where $e$ is the permittivity of free space, $x=r, d$ is the capacitance initial air gap, $w=w \max , t=$ theta, and $a$ and $b$ are the major and minor radii respectively.

Appendix B: Calculations for the parasitic capacitance and its surface areas

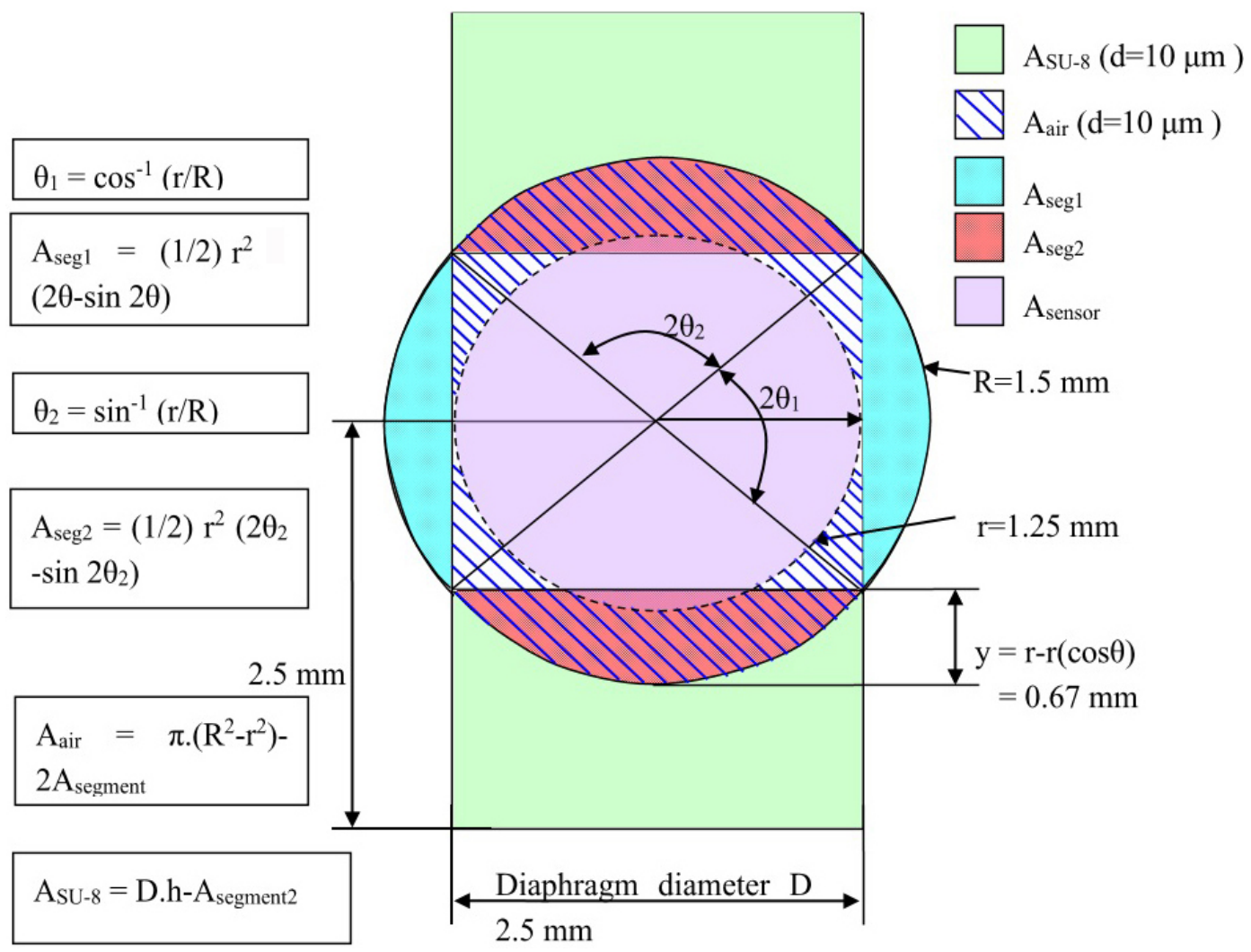

Figure B1.

$C_{p}=C_{\mathrm{SU}-8}+C_{\text {air }}+=\varepsilon_{\text {freespace }} \varepsilon_{\mathrm{SU}-8}\left(A_{\mathrm{SU}-8} / 10 \mu \mathrm{m}\right)+\varepsilon_{\mathrm{air}}\left(A_{\text {air }} / 10 \mu \mathrm{m}\right)=19.59 \mathrm{pF}$

$C_{\text {sensor }}=\varepsilon_{\text {air }}\left(A_{\text {air }} / 2 \mu \mathrm{m}\right)=21.7 \mathrm{pF}$

$C_{o}=C_{\text {sensor }}+C_{p}=41.3 \mathrm{pF}$

\footnotetext{
${ }^{5}$ Wolfram, available at: http://integrals.wolfram.com/index.jsp, last access: 12 April 2012.
} 
Acknowledgements. The author would like to show his appreciation for all research and staff members at Mechanical Engineering for their valuable assistance and guidance towards accomplishing this project.

Edited by: R. Kirchner

Reviewed by: two anonymous referees

\section{References}

A Measurement Specialties company Sensoric, Miniature SMD pressure sensor, Series Datasheet, available at: http://www. nickbelsondesign.co.uk/MS5401-AM.pdf (last access: 18 April 2012), 2009.

APOLLO: Intelligent Tyre Systems - State of the Art and Potential Technologies, IST-2001-34372, Deliverable D7, 2003.

APOLLO: Final Report, Intelligent Tyre for Accident-Free Traffic, Technical Research Centre of Finland (VTT), IST-2001-3437, Deliverable 22/23, 2005.

Bao, M. H.: Micro mechanical transducers: pressure sensors, accelerometers, and gyroscopes, Elsevier, Amsterdam, the Netherlands, 2000.

Barlian, A. A., Park, W. T., Mallon, J. R., Rastegar, A. J., and Pruitt, B. L.: Review: Semiconductor Piezoresistance for Microsystems, Proceedings of the IEEE, 97, 513-552, 2009.

Beeby, S., Ensell, G., Kraft, M., and White, N.: MEMS Mechanical Sensors, Artech House Publishers, Norwood, MA, USA, 2004.

Bever, T., Kandler, M., and Valldorf, J.: Solutions for tire pressure monitoring systems, 7th Int. Conf. Adv. Microsyst. Automot. Appl., 22-23 May 2003, Teltow/Berlin, Springer, Berlin, Germany, 2003.

Boyd, E. J. and Uttamchandani, D.: Measurement of the Anisotropy of Young's Modulus in Single-Crystal Silicon, J. Microelectromech. S., 21, 243-249, 2012.

Bracke, W., Puers, R., and Hoof, C. V.: Ultra Low Power Capacitive Sensor Interfaces, Springer, Dordrecht, the Netherlands, 2007.

Brusarosco, M., Cigada, A., and Manzoni, S.: Experimental investigation of tyre dynamics by means of MEMS accelerometers fixed on the liner, Vehicle Syst. Dynam., 46, 1013-1028, 2008.

Brusarosco, M., Cigada, A., and Manzoni, S.: Measurement and analysis of tyre and tread block dynamics due to contact phenomena, Vehicle Syst. Dynam., 49, 855-869, 2010.

Butler, L.(VK5BR) - A Dip Meter Using the Lambda Negative Resistance Circuit - Amateur Radio, January 1997, available at: http://users.tpg.com.au/users/ldbutler/NegResDipMeter. htm (last access: 30 December 2015), 1997.

Cavalloni, C., Berg, J., Krueger, S., and Gessner, W.: Overview: Principles and Technologies for Pressure Sensors for Automotive Applications, in: Advanced Microsystems for Automotive Applications Yearbook 2002, edited by: Krueger, S. and Gessener, W., Springer, Berlin-Heidelberg, Germany, 232-276, 2002.

Chang, S.-P. and Allen, M. G.: Demonstration for integrating capacitive pressure sensors with read-out circuitry on stainless steel substrate, Sensor. Actuat. A-Phys., 116, 195-204, 2004.

Chien-Hung, W., Zorman, C. A., and Mehregany, M.: Fabrication and testing of bulk micromachined silicon carbide piezoresistive pressure sensors for high temperature applications, IEEE Sens. J., 6, 316-324, 2006.
Chin, T.-L., Irving, P. Z., Oppenheim, J., and Greve, D. W.: Surface acoustic wave devices for wireless strain measurement, Sensors and Smart Structures Technologies for Civil, Mechanical, and Aerospace Systems 2010, Masayoshi Tomizuka, SPIE, 2010.

Chua, L., Juebang, Y., and Youying, Y.: Bipolar - JFET - MOSFET negative resistance devices, IEEE T. Circuits Syst., 32, 46-61, 1985.

Cullinan, M. A., Panas, R. M., DiBiasio, C. M., and Culpepper, M. L.: Scaling electromechanical sensors down to the nanoscale, Sensor. Actuat. A-Phys., 187, 162-173, 2012.

Dixon, B., Kalinin, V., Beckley, J., and Lohr, R.: A Second Generation In-Car Tire Pressure Monitoring System Based on Wireless Passive SAW Sensors, International Frequency Control Symposium and Exposition, June 2006, Miami, FL, USA, 374-380, 2006.

Druck: Resonant Pressure Transducer, RPT Series Datasheet, available at: http://www.druck.com/, last access: 19 January 2012.

Eaton, W. P. and Smith, J. H.: Micromachined pressure sensors: review and recent developments, Smart Mater. Struct., 6, 530-539, 1997.

Eddy, D. S. and Sparks, D. R.: Application of MEMS technology in automotive sensors and actuators, Proceedings of the IEEE, 86, 1747-1755, 1998.

Feng, R. and Farris, R. J.: Influence of processing conditions on the thermal and mechanical properties of SU8 negative photoresist coatings, J. Micromech. Microeng., 13, 80-88, 2003.

Fonseca, M. A.: Polymer/ceramic wireless mems pressure sensors for harsh environments: high temperature and biomedical applications, PhD, Georgia Institute of Technology, Atlanta, GA, USA, 2007.

Fonseca, M. A., English, J. M., von Arx, M., and Allen, M. G.: Wireless micromachined ceramic pressure sensor for hightemperature applications, J. Microelectromech. S., 11, 337-343, 2002.

Fragiacomo, G.: Micromachined capacitive pressure sensor with signal conditioning electronics, $\mathrm{PhD}$, Technical University of Denmark, Copenhagen, Denmark, 2012.

Frank, R.: Understanding smart sensors, Artech House, Norwood, MA, USA, 2000.

Gad-el-Hak, M.: The MEMS handbook, CRC Press LLC, FL, USA, 2002.

Greenwood, J. C.: Etched silicon vibrating senso, J. Phys. E Sci. Instrum., 17, 650-652, 1984.

Grossmann, R.: Wireless measurement of tire pressure with passive quartz sensors, P. Soc. Photo-Opt. Ins., 3670, 214-222, 1999.

Guerin, L. J.: The SU8 homepage, available at: http://www.oocities. org/guerinlj/, last access: 10 July 2008.

Halbo, L. and Ohlckers, P.: Electronic Components, Packaging and Production, University of Oslo, Oslo, Norway, 1995.

Hannan, M. A., Hussain, A., Mohamed, A., and Samad, S. A.: TPMS Data Analysis for Enhancing Intelligent Vehicle Performance, J. Appl. Sci., 8, 1926-1938,

Hew, Y., Deshmukh, S., and Huang, H.: A wireless strain sensor consumes less than $10 \mathrm{~mW}$, Smart Mater. Struct., 20, 10, 2011.

Hopcroft, M. A., Nix, W. D., and Kenny, T. W.: What is the Young's Modulus of Silicon?, J. Microelectromech. S, 19, 229-238, 2010.

Hsu, T.-R.: MEMS \& Microsystems: Design, Manufacture, and Nanoscale Engineering, John Wiley \& Sons Inc., Hoboken, NJ, USA, 2008. 
Kalinin, V.: Passive wireless strain and temperature sensors based on SAW devices, Radio and Wireless Conference, IEEE, 2004.

Kolle, C., Scherr, W., Hammerschmidt, D., Pichler, G., Motz, M., Schaffer, B., Forster, B., and Ausserlechner, U.: Ultra lowpower monolithically integrated, capacitive pressure sensor for tire pressure monitoring, Sensors, 2004, Proceedings of IEEE, 2004.

Kubba, A. and Jiang, K.: Efficiency Enhancement of a CantileverBased Vibration Energy Harvester, Sensors, 14, 188-211, 2013.

Kubba, A. and Jiang, K.: A Comprehensive Study on Technologies of Tyre Monitoring Systems and Possible Energy Solutions, Sensors, 14, 10306-10345, 2014.

Lee, Y. S. and Wise, K. D.: A batch-fabricated silicon capacitive pressure transducer with low temperature sensitivity, IEEE T. Electron. Dev., 29, 42-48, 1982.

Li, T., Hu, H., Xu, G., Zhu, K., and Fang, L.: Pressure and Temperature Microsensor Based on Surface Acoustic Wave in TPMS, Acoustic Waves, D. Dissanayake, InTech, 341-357, 2010.

Li Sainan, L. T., Wei, W., Yingping, H., Tingli, Z., and Jijun, X.: A novel SOI pressure sensor for high temperature application, Journal of Semiconductors, 36, 14014-14018, 2015.

MarketsandMarkets: Pressure Sensor Market - Global Forecast to 2020, Vancouver, WA, USA, 2015.

Muhammad, H. B.: Development of a bio-inspired MEMS based tactile sensor array for an artificial finger, $\mathrm{PhD}$, University of Birmingham, Birmingham, UK, 2012.

Nwagboso, C.: Automotive Sensory Systems, Springer, Dordrecht, the Netherlands, 2012.

Oh, J.-G., Choi, B., and Lee, S.-Y.: SAW based passive sensor with passive signal conditioning using MEMS A/D converter, Sens. Actuat. A-Phys., 141, 631-639, 2008.

Pang, C., Zhao, Z., Du, L., and Fang, Z.: Adhesive bonding with SU-8 in a vacuum for capacitive pressure sensors, Sens. Actuat. A-Phys., 147, 672-676, 2008.

Paul, C. R.: Inductance: Loop and Partial, John Wiley \& Sons Inc., Hoboken, NJ, USA, 2010.

Petersen, K. E.: Silicon as a mechanical material, Proceedings of the IEEE, 70, 420-457, 1982.

Pohl, A., Ostermayer, G., Reindl, L., and Seifert, F.: Monitoring the tire pressure at cars using passive SAW sensors, Ultrasonics Symposium, 5-8 October 1997, Toronto, ON, Canada, 1, 471474, 1997.

Pohl, A., Springer, A., Reindl, L., Seifert, F., and Weigel, R.: New applications of wirelessly interrogable passive SAW sensors, IEEE MTT-S., 7-12 June 1998, Baltimore, MD, USA, 2, 503506, 1998.

Reindl, L., Scholl, G., Ostertag, T., Ruppel, C. C. W., Bulst, W. E., and Seifert, F.: SAW devices as wireless passive sensors, Ultrasonics Symposium, 3-6 November 1996, San Antonio, TX, USA, 1, 363-367, 1996.
Rudolf, S. S. F. and Hoogerwerf, A.: Components for batterypowered wireless tire pressure and temperature monitoring systems, in Proc. SensorExpo, Detroit, USA, 15-20, 1997.

Schimetta, G., Dollinger, F., and Weigel, R.: A wireless pressuremeasurement system using a SAW hybrid sensor, IEEE T. Microw. Theory., 48, 2730-2735, 2000.

Suster, M., Young, D. J., and Ko, W. H.: Micro-power wireless transmitter for high-temperature MEMS sensing and communication applications, The Fifteenth IEEE International Conference on Micro Electro Mechanical Systems, 24 January 2002, Las Vegas, NV, USA, 641-644, 2002.

Suster, M., Ko, W. H., and Young, D. J.: Optically-powered wireless transmitter for high-temperature MEMS sensing and communication, TRANSDUCERS, 12th International Conference on Solid-State Sensors, Actuators and Microsystems, 8-12 June 2003, Boston, MA, USA, 2, 1703-1706, 2003.

Swett, D. W.: Apparatus and method for generating broad bandwidth acoustic energy, US Patent 0069708A1, 2012.

Technavio: Global Tire Pressure Monitoring System (TPMS) Market 2015-2019, London, UK, 68 pp., 2015.

Thurau, J. and Ruohio, J.: Silicon Capacitive Absolute Pressure Sensor Elements for Battery-less and Low Power Tire Pressure Monitoring, Advanced Microsystems Automotive Applications, Springer, New York, USA, 2004.

Tian, B., Zhao, Y., Jiang, Z., Zhang, L., Liao, N., Liu, Y., and Meng, C.: Fabrication and Structural Design of Micro Pressure Sensors for Tire Pressure Measurement Systems (TPMS), Sensors, 9, 1382-1393, 2009.

Timoshenko, S.: Theory of plates and shells, McGraw-Hill, New York, USA, 1940.

Tsujiuchi, N., Koizumi, T., Oshibuchi, A., and Shima, I.: Rolling Tire Vibration Caused by Road Roughness, SAE Technical papers, Grand Traverse, MI, USA, 2005.

Wang, Q. and Ko, W. H.: Modeling of touch mode capacitive sensors and diaphragms, Sens. Actuat. A-Phys., 75, 230-241, 1999.

Xiangwen, Z., Zhixue, W., Leifu, G., Yunfeng, A., and Feiyue, W.: Design considerations on intelligent tires utilizing wireless passive surface acoustic wave sensors, Fifth World Congress on Intelligent Control and Automation, 15-19 June 2004, Hangzhou, China, WCICA, 4, 3696-3700, 2004.

Yurish, S. Y. and Gomes, M. T. S. R.: Smart sensors and MEMS, Kluwer Academic in cooperation with NATO Scientific Affairs Division, Dordrecht, the Netherlands, 2005.

Zhang, X., Wang, F., Wang, Z., Wei, L., and He, D.: Intelligent tires based on wireless passive surface acoustic wave sensors, The 7th International IEEE Conference on Intelligent Transportation Systems, 3-6 October 2004, Washington DC, USA, 960-964. 2004. 